\title{
Le Club anticorps de la Societé française d'immunologie
}

\section{Structurer les atouts de la recherche française}

Lorsqu'a été publié, en 2009, le premier numéro thématique «Anticorps monoclonaux en thérapeutique » par médecine/sciences, le Groupement de recherche (GDR) CNRS 3260 «Anticorps et ciblage thérapeutique » venait d'être créé. C'était la seconde initiative de ce type (un précédent GDR, le GDR CNRS 2352, intitulé «Immuno-ciblage des Tumeurs »l'avait précédé de 2005 à 2008 et avait rassemblé 16 équipes de chercheurs impliqués dans la recherche et le développement d'anticorps, posant les bases de ce nouveau GDR) visant à structurer spécifiquement les acteurs français, malgré des moyens limités obtenus pour son animation. Actif jusqu'en 2016, sous la direction d'Hervé Watier, ce groupement de recherche rassemblait, deux fois par an, chercheurs académiques et venant de l'industrie, représentant des expertises complémentaires. En 2012, le LabEx (laboratoire d'excellence) MAblmprove, issu de ce GDR, a été financé par le Programme d'investissements d'avenir [1]. Bien que plus restreint dans son périmètre (équipes académiques de Tours et de Montpellier uniquement), le LabEx a poursuivi et relayé ce travail de structuration sur les deux sites, de 9 équipes en 2012 à 28 aujourd'hui ! En capitalisant sur sa complémentarité avec le GDR et sur sa capacité à apporter des moyens, notamment humains, MAbImprove s'est attaché à promouvoir la visibilité internationale du « réseau anticorps » français, via l'organisation d'Assises industrielles (aujourd'hui Antibody Industrial Symposia ${ }^{1}$ ) depuis 2013 [2-4], alternativement à Tours et Montpellier, permettant de rassembler les experts du secteur, académiques et privés, avec des invités internationaux prestigieux. Ce travail d'identification, d'attraction et d'animation du réseau anticorps français s'est poursuivi en étroite interaction avec la filière industrielle de l'immunothérapie MabDesign, également financée par le programme d'investissements d'avenir depuis fin 2014.

Néanmoins, il était nécessaire de pallier la fin du GDR en 2016, notamment pour les académiques hors LabEx. Le fait qu'un nombre croissant d'anticorps thérapeutiques soient dirigés contre une cible immunologique

${ }^{1}$ Site internet de MAblmprove : http://mabimprove.univ-tours.fr/fr/assise-industrielle/

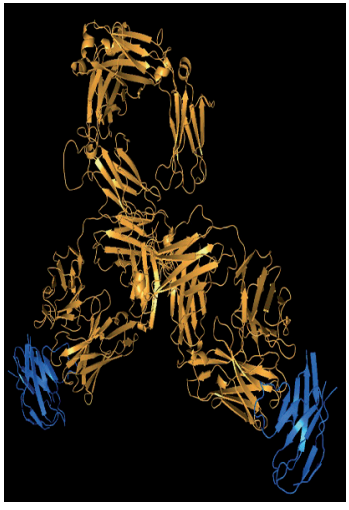

${ }^{1}$ LabEx MAbImprove, Université de Tours, 10 boulevard Tonnellé, BP 3223, 37032 Tours Cedex 1, France.

${ }^{2}$ Président de la Société française d'immunologie et directeur du Centre de recherches sur l'inflammation, Inserm U1149 et CNRS ERL8252 LabEx Inflamex, Université Paris Diderot, Paris, France.

melusine.lariviere@univ-tours.fr

(CD20, interleukines, plus récemment CTLA-4, PD-1 et PD-L1) et que leur utilisation en clinique soulève des questions fondamentales a rendu encore plus indispensable un rapprochement avec les spécialistes de l'immunologie. C'est ainsi que le « réseau anticorps » français s'est structuré au sein de la Société Française d'Immunologie (SFI) en 2017, à l'initiative de son président, Hans Yssel, avec la création du Club anticorps ${ }^{2}$.

En novembre 2017, le congrès annuel de la SFI a accueilli la réunion de mise en place de ce club, en même temps qu'était organisée une séance plénière sur le thème des immunoglobulines : "Old is new again, the moving field of immunoglobulins ». Paul Parren (Genmab) y a présenté la résolution récente de la structure des hexamères d'immunoglobuline $\mathrm{G}$ (IgG) liés au premier composant du complément (le Clq) $[5,6]$, apportant un nouvel éclairage sur la physiologie et de nouvelles perspectives en thérapie. Giovanna Scapin (Merck Sharp and Dohme) a ensuite discuté la relation structure-fonction des IgG4 et l'influence de la mutation S228P sur le phénomène de Fab-arm exchange ${ }^{3}$, au regard de la résolution cristallographique du pembrolizumab en format entier [7]. Enfin, Valérie Gouilleux-Gruart (université de Tours) a proposé un tour d'horizon des multiples rôles physiopathologiques du récepteur néonatal FcRn (neonatal Fc receptor), allant de son affinité préférentielle pour certains allotypes d'immunoglobulines

${ }^{2}$ Voir la page du club www.sfi-immunologie.com. fr/pages $/$ ?page $=785$ \&idl=21

${ }^{3}$ Les IgG4 peuvent échanger leurs chaînes lourdes de façon aléatoire avec d’autres IgG4, conduisant à la formation d'IgG4 ayant deux bras (Fab) reconnaissant des antigènes différents, soit une molécule bispécifique avec deux Fab distincts. 
et ses conséquences pour la thérapie [8] jusqu'à l'influence de son niveau d'expression sur le pronostic des cancers [9].

En 2018, les premières journées du Club anticorps se sont tenues à Paris et ont rassemblé plus de 120 personnes sur deux jours, avec des thématiques aussi variées que l'isolement et l'immortalisation de lymphocytes $B$ rares, le développement des anticorps conjugués, les modèles animaux dans les essais précliniques et l'immunomonitoring clinique lors du traitement par anticorps. Ce premier événement a accueilli des chercheurs français et étrangers, prestigieux ou prometteurs, ainsi que des présentations courtes d'étudiants et des posters ${ }^{4}$. La session d'ouverture a vu la présentation par le Pr Antonio Lanzavecchia de nouveaux mécanismes de diversification des immunoglobulines par l'insertion de séquences LAIR-1 (leukocyte-associated immunoglobulin like receptor 1 ) dans la boucle CDR3, conduisant à la sélection d'anticorps neutralisants à large spectre chez les patients atteints du paludisme [10]. Le Pr Michel Cogné a ensuite exposé des travaux récents sur les mécanismes de recombinaisons suicides lors de la commutation de classe dans les lymphocytes $B^{5}$. Les exposés et échanges entre participants se sont poursuivis pendant deux jours, témoignant d'une réelle attente des acteurs français du domaine, chercheurs, biologistes, cliniciens, académiques et industriels.

$\varepsilon$ n résumé, le Club anticorps se veut à la fois le reflet du dynamisme de la recherche française, un vecteur de partenariats avec les collègues en Europe et dans le monde et un lieu de connexions avec le tissu industriel, appelé à soutenir les ambitions de la recherche hexagonale. $\diamond$ The Antibody Club of the French Society of Immunology: structuring the assets of French research

\section{LIENS D'INTÉRÊT}

Les auteurs déclarent n'avoir aucun lien d'intérêt concernant les données publiées dans cet article.

\section{RÉFÉRENCES}

1. Pèlegrin A, Daguet A, Watier H. MAbImprove. mAbs 2014 ; 6 : 803-4.

2. Guillon-Munos A, Daguet $A$, Watier H. Antibody biosimilars: fears or opportunities? mAbs $2014 ; 6: 805-9$.

3. Bodier-Montagutelli $\varepsilon$, Respaud R, Watier H, et al. MAbdelivery: administration routes for antibody therapy Third LabEx MAbImprove industrial workshop, July 2, 2015 Tours, France. mAbs 2017 ; 9 : 579-85.

4. Martin C, Kizlik-Masson C, Pèlegrin A, et al. Antibody-drug conjugates: design and development for therapy and imaging in and beyond cancer, LabEx MAblmprove industrial workshop, July 27-28, 2017, Tours, France. $m A$ bs $2018 ; 10: 210-21$.

5. Diebolder CA, Beurskens FJ, Jong RN de, et al. Complement is activated by igg hexamers assembled at the cell surface. Science $2014 ; 343: 1260-3$.

6. Ugurlar D, Howes SC, Kreuk B-J de, et al. Structures of Cl-IgGl provide insights into how danger pattern recognition activates complement. Science $2018 ; 359: 794-7$.

7. Scapin G, Yang X, Prosise WW, et al. Structure of full-length human anti-PDI therapeutic IgG4 antibody pembrolizumab. Nat Struct Mol Biol $2015 ; 22$ 953-8.

8. Ternant $D$, Arnoult $C$, Pugnière $M$, et al. IgGl allotypes influence the pharmacokinetics of therapeutic monoclonal antibodies through $\mathrm{FcRn}$ binding. J Immunol 2016; 196 : 607-613.

9. Dalloneau $\varepsilon$, Baroukh N, Mavridis K, et al. Downregulation of the neonatal Fc receptor expression in non-small cell lung cancer tissue is associated with a poor prognosis. Oncotarget $2016 ; 7: 54415-29$.

10. Pieper K, Tan J, Piccoli L, et al. Public antibodies to malaria antigens generated by two LAIRl insertion modalities. Nature 2017 ; 548 : 597-601. ${ }^{4}$ Pour télécharger le programme complet : https://cl.assets-cdn.io/event/3497/assets/8458398194-
d2ae33434d.pdf

${ }^{5}$ Article en révision.
TIRÉS À PART

$M$. Larivière

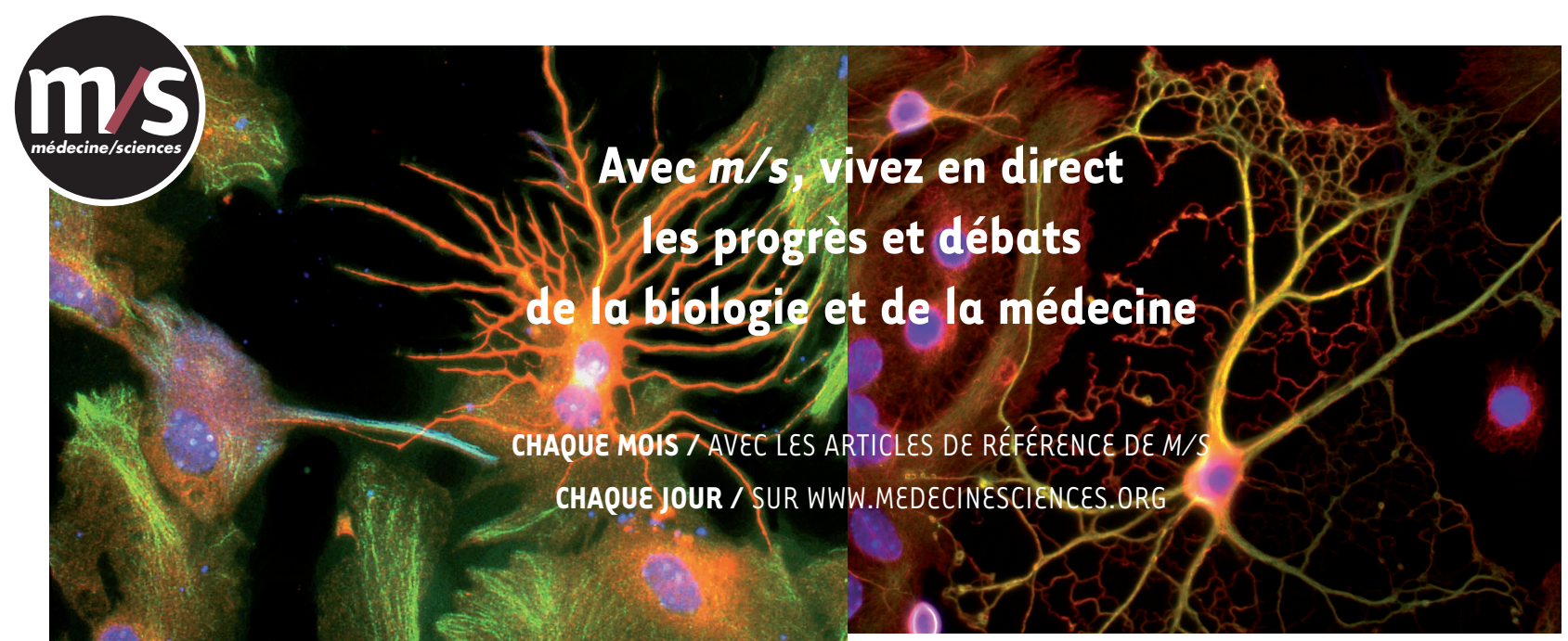

Abonnez-vous sur

www.medecinesciences.org 